\title{
36 neue Fälle von Cervicalfisteln des Uterus (28 vesicale und 8 vaginale Fisteln).
}

\author{
Nachtrag zu der „Casuistik von 165 Vesico-uterinfisteln $u$. s. w. \\ bis zum Jahre 1889" \\ (siehe dieses Archiv, Bd, XXXIII, XXXIV, XXXV).
}

Mitgetheilt von

\author{
Dr. med. Franz Ludwig Neugebauer, \\ Ordinator der gyniatrischen Univeraitätsklinik im Spital "Zum Heiligen Geist" \\ in Warschau.
}

Im vergangenen Jahre habe ich im Anschlusse an 29 Fälle von Vesico-uterinfisteln aus der Casuistik meines verstorbenen Vaters das in der Literatur vorhandene einschlägige Material von insgesammt 165 Fällen zusammengestellt und in einem Anhange zu der in diesem Archive veröffentlichten Arbeit 13 Fälle von Fistula cervicis uteri laqueatica (L. Neugebauer) beschrieben. Ich werde heute in einem Nachtrage das seit jener Zeit gesammelte neue Material kurz erörtern und zunächst noch einige Fälle von Vesico-uterinfisteln hinzufügen.

Fall 166. Vesico-uterinfistel. Eine sehr interessante Beobachtung schildert Albers in seinen: „Erläuterungen zu dem Atlas der pathologischen Anatomie" (IV. Abth., S. 807. Bonn 1847-1857): "Harnblasen-Gebärmutterfisteln." Albers schreibt: „Wenn man jene Krankheiten des Gebärmutterhalses abrechnet, welche zur Durchbohrung der Scheide und der Harnblase Anlass werden, so kommt diese Verbindung nur zwischen der schwangeren Gebärmutter und Harnblase zu Stande. Es ist wahrscheinlich der Brand, welcher die Verbindung zwischen beiden Theilen vermittelt. Der Fall, welcher diese Krankheit nicht allein nachweist, sondern auch über die Art und Weise ihres Entstehens Aufschluss giebt, ist von Ferras (Bulletin des Sciences médicales 1828, Juillet) aufgezeichnet. Bei einer im siebenten Monate Schwangeren stellten sich die $\mathrm{Zu}$ fälle der heftigsten Entzündung des Unterleibes ein; bald darauf gingen einige Fötusknochen und zersetzte thierische Materie durch 
die Harnröhre ab. Bei der Leichenöffnung fand man die vordere Wand der Gebärmutter und die hintere Wand der Harnblase durch brandige Zerstörung beseitigt und beider Organe Höhlen in Verbindung. In der Harnblase fand man noch viele Fötusknochen und ausserdem einen Spulwurm." (Im Anschlusse hieran erwähnt Albers noch eine Beobachtung, die Dr. Trimborn in Godesberg an einer Kranken machte, bei welcher es jedoch nicht ausgeschlossen ist, dass nicht vielmehr ein Durchbruch einer: Extrauterinschwangerschaft vorlag, - zur Section kam es nicht, weil die Frau glücklicherweise am Leben blieb). Im Vergleiche zu der gewöhnlichen Aetiologie der Vesico-uterinfisteln ist die obige Beobachtung wohl ziemlich alleinstehend, die Ursache zu jenem Durchbruche wahrscheinlich Incarceration der schwangeren Gebärmutter.

Fall 167, von M ünchmeyer beobachtet (,Ueber die Entbindungen mittels der Zange an der Königl. Frauenklinik in Dresden." dieses Archiv, Bd. XXXVI, Hft. 1, S. 23, Fall 13): „Frau Knospe (Journ.-Nr. 617), 37jährige I para mit allgemein verengtem platten Becken. Conjugata vera $8,5 \mathrm{~cm}$. Schädellage, Perforation, Kranioklasie, manuelle Placentarlösung, Collumriss, grosser Scheidenriss. Kind $2700 \mathrm{~g}, 50 \mathrm{~cm}$ lang, Knabe. Vom 6. bis 12. Tage Fieber, Blasencervicalfistel, Lähmung der Beinnerven; verlegt am 40. Tage.“

Fall 168-169, von Bezdziek beschrieben („Ueber die in der Zeit von Ostern 1886 bis Ostern 1889 in der Universitäts-Frauenklinik zu Breslau operirten Urogenitalfisteln." Inaugural-Dissertation. Breslau 1889. S. 38-40):

1) Susanne St., 40 jährig (Nr. 110, 1886/7); in 20 jähriger Ehe fünf Mal natürlich entbunden, zuletzt vor fünf Wochen. Geburtsarbeit von Montag Abend bis Mittwoch früh, lebendes Mädchen. Incontinenz vom nächsten Tage an, wie es der Kranken schien, sicher festgestellt aber erst nach drei Wochen, als die Kranke das Bett verliess. Am 1. Februar 1887, nach rechtsseitiger Spaltung des Collum, Anfrischung and Naht, Drain in die Blase und Jodoform in die Scheide. Täglicher Verbandwechsel, vom sechsten Tage an Harnträufeln durch die Scheide. Am 27. Februar Nähte entfernt, vergebliche Aetzung mit Lapis. Nachoperation am 21. März 1887: Anfrischung senkrecht zur ersten, Seidennaht, kein Drain in die Blase. Die Kranke hält den Harn und wird mit liegenden Fäden geheilt entlassen am 29. März.

2) Rickel W. aus Zduńska Wola in Polen (Nr. 62, 1888/9), 23jäbrig, aufgenommen am 5. Mai 1888. Vor sieben Wochen Forceps bei todtem Kinde. Rhachitische Person. Muttermund lässt Zeigefinger ein, die scharfrandige Fistel lässt denselben frei in die Blase eindringen. Anfrischung und Naht am 12. Juni 1888, Drain, Jodoformgaze. Achttägiges Fieber, nur theilweise Heilung. Nachoperation am 5. Juli 1888. Geheilt entlassen am 12. Juli 1888. 
Fall 170-171. Je einen weiteren Fall haben durch unmittelbaren Nahtverschluss geheilt laut mündlicher Mittheilung Dr. Matlakowski und Prof. Jastreboff in Warschau in den Jahren 1889 und 1890.

Fall 172-174. Prof. Olshausen hat laut mündlicher Mittheilung drei weitere Fälle geheilt.

Fall 175-177. Einen fünften von Winckel geheilten Fall, sowie einen von Winckel angeführten Fall vou Wilms erwähnt Frl, Philim on ow a in ihrer Arbeit; einen siebenten erwähnt W inckel selbst (s. L.-V. Nachtrag).

Fall 178. Einen weiteren Fall aus Leipzig beschreibt Heyder (Verhandlungen der Geburtshülflichen Gesellschaft in Leipzig vom 17. Juni 1889, siehe Centralblatt für Gynäkologie 1890 , Nr. 19, S. 344): „Das Bemerkenswerthe dieses Falles bestand darin, dass an Stelle der verloren gegangenen Vaginalportion innerhalb narbigen $\mathrm{Ge}$ webes des Cervixstumpfes, kaum $4 \mathrm{~mm}$ von einander entfernt, 2 Oeffnungen sich fanden, von denen die eine in die Cervix, die andere, rechts von ihr gelegen, in die Blase mündete. Trotz der gegenseitigen Nähe beider Mündungen gelang es, die Fistel direct anzufrisehen und durch Naht zu schliessen, und zwar durch die von Sänger schon in einem früheren Falle angewandte und beschriebene Erweiterung des stenosirten Cervicalcanales durch Laminaria, tiefe laterale Spaltung der linken Cervixwand und Nahtumsäumung der Spaltränder, wodurch so viel Platz gewonnen wurde, dass die Anfrischung genuigend hoch in die rechte seitliche Cervicalwand hinauf verlegt werden konnte, wodurch die jetzt breite Cervixmündung eine quer-trichterförmige Gestalt bekam. Es wurde dadurch beiden Indicationen: Verschluss der Fistel und Herstellung eines weiten Cervicalcanales genügt."

Fall 179. Einen nach dem Vorgange Follet's und Wölfler's geheilten Fall theilt Champneys mit (laut Schmidt's Jahrbüchern, Bd. 227, Nr. 7, S. 52; berichtet nach: Transactions of the Obstetrical Society of London, Vol. XXX for the year 1888, p. 348): 38 jährige Frau, vier Mal wegen Beckenenge schwer entbunden, litt drei Tage nach der letzten Entbindung (Zange) an Harnträufeln. Die Fistel sass rechts im Cervicalcanale, ungefähr $1,4 \mathrm{~cm}$ vom Orificium externum entfernt. Champneys durchtrennte das vordere Scheidengewölbe quer, wie bei der Totalexstirpation des Uterus, löste die Blase $a b$ und legte so die Fistelöffnung ganz frei. Dieselbe, für den Mittelfinger durchgängig, wurde in der Blasenwand durch sieben feine Silberdrähte, in der Cervicalwand durch gleiche vier geschlossen und dann der Wundrand des Scheidengewölbes vereinigt durch vier Nähte mit dem vorderen Muttermundssaume. In ähnlichen Fällen, heisst es weiter, werde Champneys Chromsäurecatgut oder Silkworm nehmen, hier aber habe er der Neuheit des Vorgehens halber ein völlig zuverlässiges Nahtmaterial vorgezogen.

Fall 180. An derselben Stelle ist anch folgender ganz eigenartige Fall von J. Cullingworth (a. a. O., p. 320, Vol. XXXI) be- 
schrieben: 37 jährige Frau, drei Mal durch Kunsthülfe entbunden; das erste und dritte Kind wurden zerkleinert, das zweite mittels der Zange entwickelt. Harnfluss vom ersten Tage nach der Entbindung ab. Es zeigte sich eine Blasenscheidenfistel, welche die Fingerspitze durchliess, unmittelbar quer vor der Cervix und höher oben noch eine zweite zwischen Cervicalwand und Blase. Cullingw orth spaltete am 14. Februar 1889 die vordere Lippe bis zur letzteren Fistel und vereinigte die angefrischten Flächen durch vier Silkwormnähte, deren Mehrzahl in der Blase geknüpft wurde, und leitete die lang gelassenen Fäden durch die weiter vorn gelegene Fistel heraus. Am 13. Tage Entfernung der Nähte, Fistel geschlossen. Am 29. März wurde die Blasenscheidenfistel durch Silkworm geschlossen, am achten Tage die Nähte entfernt. Cullingworth giebt an, wenn die Vereinigung der cervico-vaginalen Fistel auf diese Weise nicht gelungen wäre, so hätte er nach Champneys die Cervix von der Blase abgetrennt und von der Wunde des Scheidengewölbes aus genäht.

Fall 181 u. 182. Ferner hatte im Jahre 1890 lant mündlicher Mittheilung Prof. Jastreboff in Warschau noch 2 Naturheilungen von Blasencervicalfisteln zu verzeichnen.

Fall 183. Engström entdeckte nach einer schwierigen dritten Geburt bei plattem Becken, wo acht 'Tage nach der Geburt Harntiäufeln sich zeigte, eine kleinfingergrosse Verbindung zwischen Gebärmutterhals und Blase, die sich im Laufe des Wochenbettes wesentlich verkleinerte. Fünf Wochen nach der Geburt Operation. Vernähung mit Juniperuscatgut in zwei Etagen von der Scheide bezw. Cervix aus mit primärer Heilung.

Fall 184. Nach Prof. Stumpf's Bericht in Frommel's Jahresbericht für 1889 erzielte Vincenzo eine Heilung durch operativen Nahtverschluss bei einer hochsitzenden, nach Perforation nnd Kranioklasie bei allgemein verengtem Becken entstandenen Fistel, deren uterine Mündung trotz tiefer lateraler Spaltung der Cervix, die sechs Tage vor der eigentlichen Fisteloperation ausgefiihrt wurde, nicht freigelegt werden konnte. Die Art der Operation ist mir aus dem Berichte nicht klar geworden. Es heisst bei Stumpf (a. a. O. S. 681): „Es wurde ein Längsstreifen der Mucosa und Submucosa der vorderen Lippe angefrischt and die Wunde durch fünf Nähte quer vereinigt. An den ersten drei Tagen nach der Operation erfolgte noch spontaner Harnabgang, der dann aufhörte. Vollständige Heilung." Weiter heisst es daselbst: "In einem offenen Briefe zu dieser Publication erklärt sich Novaro gegen diese Art der Operation und befürwortet fiur solche schwer zugängliche Fisteln die Methode von Follet-Wolfler und in zweiter Linie die von Neugebauer."

Fall 185 u. 186. In Stumpf's erwähntem Berichte heisst es (S. 673): "Von den drei Fällen Johnson's betrifft einer eine Blasenscheidenfistel, bei den beiden anderen aber handelte es sich um eine Complication mit Blasencervicalfistel, weil beide Male die vordere Muttermundslippe in den Defect hineinbezogen war. In den beiden 
beschriebenen Fällen war bei der Entbindung, nach welcher die Fistel entstanden war, der Forceps angewandt worden. In dem einen Falle konnte nach langer präparatorischer Behandlung der Defect auf gewöhnliche Art zum Verschluss gebracht werden, bei dem zweiten aber wurde die hintere Muttermundslippe in den Defect eingenäht." (Waren dies wirklich reine Cervicalfisteln?)

Fall 187. Mittelhäuser beschreibt folgenden Fall ans der Jenaer Klinik: Frau M. E., 32jährig, verheirathet im 24. Lebensjahre, III para, hat drei Mal normal geboren, beim vierten Kinde Wendung durch die Hebamme, zuletzt natürliche Austreibung eines todten Kindes unter Krampfwehen. Am fünften Tage plötzlich Harnfluss. Wegen Schwäche zehn Wochen zu Bett, absolute Incontinenz in jeder Lage und Stellung. Beiderseitiges Colobom, mehr links, Narbe im linken Scheidengewölbe, Uterus in Retroversion, Fundus links. An der Innenseite der Vorderlippe, 1,5 bis $2 \mathrm{~cm}$ vom Rande entfernt, links die kleine runde Fistel. Operation in Steissrückenlage mit erhöhtem Steisse. Nahtschlusslinie der drei Nähte verläuft von vorn nach hinten. S Sechs Tage lang katheterisirt, dann natürliche Harnentleerung. Geheilt entlassen.

Fall 188. Tillaux beobachtete einmal gleichzeitig drei Fisteln, eine Blasenscheiden-, eine Harnröhrenscheidenfistel und eine Cervixblasenfistel und heilte die Kranke durch quere Obliteration.

Fall 189. Stumpf führt ferner eine Beobachtung von Morell Terry an, geheilt durch constante Lage und wiederholten Katheterismus.

Ausserdem sollen nach Stumpf's Berichte die Arbeiten von Heinricius und Torggler Fälle von Vesico-uterinfisteln enthalten (siehe a. a. 0.). Ich habe jedoch am angegebenen Orte keinen Fall von reiner Vesico-uterinfistel finden können, sondern nur vesico-utero-vaginale Fisteln, die wir von den Cervicalfisteln mit Absicht trennen. Nach Stumpf's Bericht sollen auch Sherwood Dunn and Mac Arthur Cervicalfisteln beobachtet haben.

Zählen wir diese beiden Fälle als Nr. 190 und 191 und fügen noch weitere 2 eigene aus der gyniatrischen Universitätsklinik meines zu früh dahingeschiedenen Vaters hinzu, so ergiebt sich bislang eine Gesammtcasuistik von 193 Fällen von Vesico-uterinfisteln, von denen 191 die Cervix betreffen, also Cervicalfisteln sind, und 2 Uteruskörperblasenfisteln (Fall 123 und 166 meiner Statistik: Déroubaix und Ferras). Von diesen 193 Fällen von Vesico-uterin- bezw. 191 Fällen von Cervico-utero-vesicalfisteln kommen nicht weniger als 31 auf die Klinik meines verstorbenen Vaters.

Unsere beiden letzten Fälle sind folgende: 
Fall 192. Sophie Ratatik, 45 jährige Dienstfrau, trat am 8./20. November 1889 ein (sub Nr. 75 II). Seit 28 .Jahren verheirathet, hat sie 11 Kinder natürlich, ausgetragen, lebend geboren, und zwar alle Kinder in Schädellage, das letzte Kind nach 48 stündiger Geburtsdauer vor elf Monaten. Die Wöchnerin bemerkte, als sie am elften 'Tage aufstand, theilweises Harnträufeln durch die Seheide. Rechtsseitiges Colobom des Muttermundes, $1 \mathrm{~cm}$ über dem Saume der vorderen Muttermundslippe eine kleine Cervicalfistel. Nach mehrfachen vergeblichen Aetzungen mit Höllenstein wurde die vordere Muttermundslippe in schräger Richtung bis in die Fistel hiuein, nach dem zuerst von meinem Vater beschriebenen Verfahren, gespalten und dann die Wunde, ein spitzwinkliges gleichschenkliges Dreieck zusammengefaltet und durch neun Kupferdrahtknopfnähte und eine Entspannungsnaht geschlossen am 25. December 1889 (6. Januar 1890). Am 21. Januar (2. Februar) 1890 geheilt entlassen.

Fal1 193. Unsere letzte gemeinschaftliche Beobachtung betraf eine von. Dr. Kos sows ki aus Nieszawa zugesandte Jiidin, Jaku bowicz, 42jährige Xpara (siehe Ambulatorisches Journal Nr. 2160 vom 10. Februar 1890). Das erste Kind kam spontan todt zur Welt, das zweite, dritte, vierte lebend von selbst, das fünfte todt mittels Zange, das sechste und siebente lebend von selbst, das neunte im achten Monate todt, das letzte in Fusslage mittels Zange am nachfolgenden Kopfe. Sofortiger Harnfluss. Hintere Lippe seitlich eingerissen; erst nachdem man die vordere Lippe stark herabgezogen, bemerkt man hinter ihr verborgen, wie hinter einer Querfalte, eine Cervicalfistel von $1^{1 / 2} \mathrm{~cm}$ Durchmesser, die grösste unter unserem Materiale. Periode regelmässig vorhanden. Die Fistel besteht trotz ihrer Grösse doch schon seit mehr als sechs Monaten. Leider wollte die Kranke auf keinen Fall auf die Kost unseres christlichen Spitals eingehen und rerliess, da eine Ausnahme von den Vorschriften nicht gestattet wird, die Ambulanz, ohne sich aufnehmen zu lassen. Sie fand, den Satzungen ibres Glaubens getreu, dass der Harnfluss ein geringeres Uebel sei, als das Bewusstsein, gegen die jüdischen Vorsehriften za handeln.

Erwähnt werden muss hier ferner der neue von Trendelenburg vorgeschlagene Weg zur operativen Heilung sonst unzugänglicher Blasenfisteln durch die Sectio alta. Dieses Verfahren wird gewiss sich das Bürgerrecht erwerben und neben den bisherigen des unmittelbaren Nahtverschlusses als ein neues hinzuzufuigen sein (,Ueber Blasenscheidenfisteloperationen und über Beckenhochlagerung bei Operationen in der Bauchhöhle." Sammlung klinischer Vorträge, herausgegeben von Richard Volkmann. Leipzig 1890. Nr. 355). Dieses sinnreich gewählte Verfahren besteht einfach in dem unmittelbaren Nahtverschlusse der Fistel von der Blase aus, nachdem durch eine Sectio alta der Zutritt eröffnet ist, und zwar bei Anwendung der bekannten und ron Leopold 
neuerdings so warm angerathenen, von Trendelenburg sehr empfohlenen abhängigen Rückenlage. Trendelenburg empfiehlt diesen Weg besonders für die schwer zugänglichen Fälle von Blasengebärmutterscheidenfisteln und die Harnleiterscheidenfisteln, vor allem aber für die Blasengebärmutterfisteln, welche seiner Ansicht nach mit dem Verfahren der unmittelbaren Vereinigung von der Scheide aus gar nicht anzugreifen seien. Letztere Ansicht ist eine falsche, aber die Unkenntniss der gyniatrischen $\mathrm{Ca}$ suistik bei einem Chirurgen gewiss verzeihlich. Diese irrige Angabe schmälert in nichts das Verdienst Trendelenburg's, so mancher leidenden Frau die Niere zu erhalten, wo man sonst einer unheilbaren Ureterfistel wegen zur Nephrektomie zu greifen sich gezwungen sah oder zur künstlichen Anlegung einer Blasenscheidenfistel und einer Kolpokleisis. An der Hand der heutigen Operationsverfahren dürfte somit gewiss die grösste Mehrzahl der Blasencervixfisteln einem unmittelbaren Nahtverschlusse zugänglich sein. Es geht aus der Veröffentlichung Trendelenburg's nicht hervor, ob er persönlich eine Vesico-uterinfistel auf diese Weise operirt hat. Die nächste Zukunft wird ja wohl praktische Belege bringen für die Begründung der neven Trendelenburg'schen Operationsmethode.

Im vorliegenden Hefte dieses Archives hat Dr. P. Ba'u mm aus Oppeln einen Aufsatz unter der Aufschrift: „Zur Operation der Blasen-Cervixfisteln von der Blase aus" veröffentlicht mit Anwendung des von Trendelenburg empfohlenen vesicalen Operationsweges. Soweit ich den Verfasser recht verstanden, handelte es sich jedoch im vorliegenden Falle nicht um eine reine Cervicalfistel, sondern um eine Fistula utero-vesico-vaginalis profunda. Baumm erkennt vollkommen die Lichtseiten des Trendelenburg'schen Verfahrens an, hat aber auch dessen Schattenseiten kennen gelernt und ist daher seine Arbeit für Alle von grossem Interesse, die so viel wie wir mit Harnfisteln zu thun haben, ich möchte sagen alle 14 Tage eine Harnfistel in Behandlung bekommen.

Wie entmuthigend auch die Erfahrungen Baumm's sein mögen, für gewisse Fälle wird sich das von Trendelenburg angegebene Verfahren gewiss Bahn brechen. Dort, wo der vaginale Weg nicht zum Ziele führt, wird der vesicale Weg immer noch dem renalen vorzuziehen sein bei Harnleiterfisteln, der Kolpokleisis bei durch die Scheide unzugänglichen anderen Harnfisteln. 
Winckel spricht sich in seinem Vortrage gegen die Sänger'sche Operationsmethode aus, durch welche der Fistelcanal an seinem unteren Ende geschlossen wird, während der Gang selbst offen bleibt, weil es dabei zur Bildung von Taschen, zum Stagniren und Zersetzung des Harnes und zu Steinbildung kommen kann. „Er zieht,“ wo dies thunlich, „directen Nahtverschluss der Fistel mit Fil de Florence vor" (Stu mpf).

Es sei mir gestattet, noch einige seit 1889 in unserer Klinik beobachtete neuere Fälle von Mutterhalsscheidengewölbefisteln (Fistula utero-cervico-vaginalis s. utero-vaginalis laqueatica) kurz zu erwähnen, darunter einen, der bezüglich seiner Aetiologie vielleicht einzig dasteht.

Fall 14 unserer gesammten Tabelle, Fal1 12 unserer Klinik: Seltener Fall von vaginaler Cervixfistel nach instrumentellem Aborte. Am 4. März 1890 wurde (unter Nr. 582; 25, $1890 \mathrm{I}$ ) die 27jährige Handwerkersfrau Ald. B. aus Pińsk in die gyniatrische Universitätsklinik aufgenommen. Menstruirt mit 15 Jahren und verheirathet seit dem 25. Jahre, bat sie doch als 24jühriges Mädchen bereits ihre erste Schwangerschaft durchgemacht. Dieselbe endigte mit einem im sechsten Monate künstlich von einem vor mehreren Jahren verstorbenen Arzte in Pinsk hervorgerufenen Aborte. Nachdem laut Aussagen der Frau eine Laminariaeinführung in die Gebärmutteröffnung nicht zum Ziele geführt, soll jener Arzt Instrumente in die Gebärmutter eingebracht haben. Näheres weiss die Kranke nicht anzugeben. Es folgte der Abort. Fünf Monate später heirathete A. B. ihren jetzigen Gatten, wurde nach fünf Mo. naten schwanger und abortirte diesmal angeblich von selbst im vierten Monate. Zehn Wochen später Blutsturz und eine Gebärmutterentzündung, die ein dreimonatiges Krankenlager zur Folge batte. Es stellte sich dabei auch Durchfall ein, der längere Zeit anbielt und mit Darmblutungen verbunden war. Seit jener Zeit Hypermenorrhoe und beständiger dumpfer Druckschmerz in der rechten Seite.

Die Untersuchung ergab folgenden seltenen Befund: Scheide weit, Uterus verlängert, besonders die lange, koniseh zugespitzte Vaginalportion, die nach hinten gerichtet erscheint bei Knieellenbogenlage. Nur mit Mühe entdeckt man den sehr engen Muttermund, der jedoch eine feine Sonde durchgehen lässt. Nachdem der Scheidentheil durch eine Muzeux'sche Zange herabgezogen ist, erblickt man ein überraschendes Bild: Einen mehr als Fingerdicke weiten Verlust der hinteren Wand der Cervix, d. b. eine grosse Fistula cervicis uteri laqueatica posterior. Die Fistel hatte eine sagittale Richtung, war ungefähr $2 \mathrm{~cm}$ lang und $1,3 \mathrm{~cm}$ breit, ein Oval mit überwiegendem Längendurchmesser. Die vordere Wand der Cervix stülpte sich convex in die Fistelöffnung der hinteren hinein, so dass zwischen den seitlichen Fistelrändern und der Fläche der Cervix der vorderen Wand der Cervix eine Art rinnenförmiger Falz bestand. Die Fistelränder 
waren ziemlich dick und auffallend hart. Die Schleimhaut der Cervix der vorderen Wand nicht geschwïrig, glatt, aber dunkelroth. Am Muttermunde eine ganz kleine Erosion von bestehendem Katarrhe her. Es gelang eine dünne Sonde, später auch eine dickere, durch den Muttermund hinein und zur Fistel hinauszuführen.

Die operative Heilung war ziemlich schwierig wegen der grossen Spannung der weit abstehenden Wundränder, desto mehr, da breit angefrischt worden war. Die Anfrischung geschah wie bei Blasenscheidenfisteln, die Vereinigungslinie der Wunde verlief schräg von links unten nach rechts oben, die Nähte wurden also senkrecht zu dieser Linie durchgeführt, so dass die spätere Narbe eine schräge Verlaufsrichtung bekam. Erst nach wiederholter Operation gelang der Verschluss der Fistel. Die Kranke wurde am 25. Mai (6. Juni) 1890 in ihre Heimath entlassen, nachdem durch zweckmässige Allgemeinbehandlung und Bäder die Kräfte gehoben und die träge Verdauung beseitigt worden war. Der lästige Ausfluss hatte sich verloren, die nächste Periode war immer noch reichlich, aber nicht besonders schmerzhaft. Das Interessanteste ist das Vorhandensein einer so grossen Fistel, ohne dass die Frau einè schwerere Geburt durchgemacht hatte.

War die grosse Fistel entstanden durch eine zufällige instrumentelle Verletzung von Seiten des Arztes oder einer anderen Person, der etwas daran lag, den Abort herbeizuführen? Oder aber entstand die Fistel nach absichtlicher Incision, wie z. B. solche gemacht wird bei einer während der Schwangerschaft erworbenen Verschliessung des Muttermundes? Oder aber entstand die Fistel infolge eines örtlichen Entzündungsvorganges, herbeigeführt durch Einkeilung und Druck? Das ist nicht wahrscheinlich im sechsten Monate der Schwangerschaft bei normalem Becken ohne Geschwülste. Als einfache Verschwärung, wie sie bei puerperalen, diphtherischen, typhösen, tuberkulösen, syphilitischen Vorgängen vorkommt? Es liegt keinerlei Anhaltspunkt für eine solche Annahme vor. Ist endlich die Cervixfistel vielleicht der Rest eines bis zum Muttermunde ursprünglich reichenden Risses der Cervix, dessen in der Muttermundslippe belegener Theil von selbst verheilte, während der obere Theil offen blieb? Auch diese Annahme entbehrt der Wahrseheinlichkeit, weil die konische Vaginalportion völlig glatt und ohne Spur von Narbe oder Wunde ist, ebenso der Muttermund nicht eingerissen, sondern jungfräulich. War eine pathologische Rigidität und Stenose des Muttermundes der Grund zu einer Cervixruptur sub abortu?

Es scheint am richtigsten, die Entstehung der Fistel auf den ersten künstlichen Abort, bez. eine instrumentelle Durchbohrung der Wand der Cervix vielleicht mit der Sonde zuriickzuführen, indem diese Verletzung in der Folge bei dem Austreten der Frucht (wahrscheinlich durch dieselbe?) noch vergrössert wurde. Aller Wahrscheinlichkeit nach ist die Frucht durch jene Fistelöffnung ausgetreten, ebenso wie die spätere zweite Schwängerung und der erneute Abort durch die Fistel hindurch stattgefunden haben mögen. Für 
letztere Annahme spricht die grosse Engigkeit des verschwollenen untersten Cervixabschnittes und Muttermundes, der, wie gesagt, nur schwer zu finden ist, so dass man auf den ersten Blick in der Fistel einen stark erweiterten und eingerissenen Muttermund vor sich za haben glaubt.

Gleiche Fälle von Perforatio cervicis bei Abortus instrumentalis dürften sich vielleicht in der Literatur finden, immerhin fand ich noch keinen einer nachgebliebenen Cervixlaquearfistel. Wahrscheinlich war der zweite Abort, wenn er wirklich von selbst erfolgte, eine Folge des durch die Fistel bedingten chronischen Cervixkatarrhes.

Ausser diesem Falle hatten wir noch folgende ätiologisch mit der erschwerten Geburt eines ausgetragenen Kindes in Verbindung stehende Fälle von Cervixlaquearfisteln seither zu verzeichnen:

Fall 15 (13) von Utero-vaginalfistel: Katharine Golen, 28jährige Banersfrau aus Grabice, seit zwei Jahren verheirathet, trat am 31. October 1889 in die Klinik ein (Nr. 3984, Nr. 803 meines Journals). Kleine rhachitische Person von $140 \mathrm{~cm}$ Höhe, Conjugata externa 15,5, Conjugata vera $6,25 \mathrm{~cm}$. I para. Am 20. Februar 1889, also vor sieben Monaten, nach dreitägiger Geburtsarbeit natürliche Austreibung eines reifen todten Kindes mit sofortigem Harnflusse durch die Scheide. Zehn Wochen lang nnter Fieber zu Bett gelegen, Regel seither schon fünf Mal aufgetreten. Die vordere Muttermundslippe ist völlig zerstört, dicht daran und in die Lippensubstanz reichend eine querliegende breite Fistula utero-vesico-vaginalis profunda, auseinandergezogen $2 \mathrm{~cm}$ breit. In der hinteren Muttermundslippe eine gleich grosse Fistel, querliegendes Oval mit einem nach dem freien Saume der hinteren Lippe spitz auslaufenden Einrisse linkerseits; es war also hier wahrscheinlich ursprünglich ein sagittaler Einriss der hinteren Lippe links seitlich, der sich dann in einen mehr queren fortsetzte. Der Längenriss heilte, der querliegende Theil der Wunde blieb unvereint. Der Schambeinknorpel ragt ungemein stark gegen die Beckenhöhle vor und ist allem Anscheine nach die Causa princeps malorum gewesen angesichts der hochgradigen Abplattung des Beckens. Ich durchschnitt am 3. (15.) November 1889 die hintere Muttermundslippe unterhalb der Fistel und schlug mittels Häkchen die Lippenhälften auseinander, um besseren Zutritt zu der oberen Umrandung der tiefen Blasengebärmutterscheidenfistel zu gewinnen und anzufrischen. Unmittelbarer Nahtverschluss der Blasenfistel. Geheilt entlassen am 4. (16.) December 1889. Die Laquearfistel liess ich vorläufig nach Spaltung jener Brïcke unbeachtet. Es könnte eine spätere Emmet'sche Operation nothwendig werden, falls Impotentia gestandi eintreten sollte.

Fall 16 (14). Rachel Markowicz (Nr. 1991) trat am 12. (24,) November 1887 in die Klinik ein. 30 jährige Händlersfrau, seit zehn Jahren verheirathet, hat die ersten drei Kinder reif, lebend, aber schwer geboren, das letzte vierte Kind vor neun Wochen todt mittels Zange (Dr. I waszkiewicz aus Końsk), 36 Stunden nach Wasserabfluss. Vom 10. Tage an Harnfluss durch die Scheide. Allgemein 
mässig verengtes plattes Becken. Fistula utero-vesico-vaginalis superficialis in der Fortsetzung eines oberhalb narbig geheilten vorderen Risses der Cervix rechterseits. Linkerseits ganz in der Ecke, in der Fortsetzung eines narbig verheilten Risses des linken Muttermundswinkels, eine linsengrosse Perforation der hinteren Muttermundslippe: Fistula cervico-utero-vaginalis laqueatica pulsilla sinistra.

Die Blasenfistel wurde mit sieben Kupferdrähten geschlossen, die Kranke am 6. (18.) December 1887 geheilt entlassen.

Fall 17 (15), Frau Emilie Dalg aus Chańsk trat am 3. November 1889 in meine Behandlung (J.-Nr. 3797/616 1889) wegen einer sehr grossen, breiten Vesico-vaginalfistel mit in den seitlichen Ecken stark narbig zurückgezogenen Rändern. Die erste Schwangerschaft endete mit Abort, die zweite ergab ein ausgetragenes todtes Kind, die dritte ein lebendes, die vierte (letzte) vor neun Woehen nach zweitägiger Geburt ein todtes Kind. Die Hebamme hat den vorausgehenden Schädel mit einem Haken herausgebracht. Ausser der Blasenfistel fand sich eine kleine Fistula laqueatica eervieis uteri sinistra ganz seitlich gelegen. Ich machte die Kolpokleisis, die jedoch mehrere Nachoperationen erforderte. Trotzdem klagt die Frau ab und zu über Nässe, obwohl sie den Harn willkürlich entleert.

Fall 18 (16). Josephine Mickiewicz, 24jährige Bauersfrau, Ipara, aus Ciechanowo in Polen, trat ein am 18. Februar 1890 (unter Nr. 2179/99) wegen einer grossen, tiefen Blasengebärmutterscheidenfistel. 52 stündige Geburtsdauer nach Wasserabfluss. Forceps, dann Kranioklasie. Zwei Wochen zu Bett. Rhachitische Kyphoskoliose mit Conjugata vera $6,5 \mathrm{~cm}$, Conjugata externa $15 \mathrm{~cm}$. Es war eine derartige Drehung der Cervix durch Narbenschrumpfung eingetreten nach Zerstörung der vorderen Muttermundslippe, dass die Vaginalportion in die Harnblase hineinsah, also eine Intussusceptio portionis vaginalis uteri vesicalis, so dass man in Knieellenbogenlage mit Kristeller's Entenschnabelspeculum untersuchend den Muttermund nicht erblickte, sondern nur die hintere Muttermundslippe und den ihr gegenüberliegenden unteren bez. vorderen Rand der Blasenfistel. Es lag also eine Neigung vor zur natürlichen Verlegung der Fistel bezw. Heilung der Incontinenz durch natürliche Metrokolpokleisis auf dem Wege der Narbenschrumpfung mit Retroversio uteri. In der hinteren frei zugängigen Lippe nun fand sich noch eine begleitende. Fistula laqueatica eervicis uteri posterior, wahrscheinlich als Folge einer puerperalen theilweisen Abquetschung der hinteren Lippe durch das stark vorspringende Promontorium.

Fall 19 (17). Endlich sei hier noch ein letzter, ebenfalls sehr interessanter Fall beschrieben, den mir College Isserson aus Biatystok zusandte. Eitla Mowszowicz, 30jährige Ipara, trat ein am 5. Mai 1890 (unter Nr. 2310/320). Rhachitische Beckenenge II. Grades. Seit zwei Jahren verheirathet, hat sie vor elf Monaten von selbst ein todtes reifes Kind geboren und von da an theilweisen Harnfluss bemerkt. Bei der ersten Betrachtung in Knieellenbogen- 
lage sieht man nirgends eine Fistel, und doch fliesst in die Blase eingespritzte Milch hervor zwisehen vorderer Muttermundslippe und Blasenscheidenwand. Erst nachdem die vordere Lippe durch zwei eingesetzte Häkchen in die Höhe gezogen wurde, entdeckte ich tief im Scheidengrunde eine ganz kleine Oeffnung. Eine feine Sonde in die Blase hier eingeführt, stiess mit dem Katheter zusammen. Zwei Aetzungen mit Höllenstein, ambulatorisch gemacht, genügten zur Heilung. Betrachtete man nun den Muttermund genauer, so ergab sich ausser einem beiderseitigen lateralen Colobom noch ein hasenschartenartiger Einriss der vorderen Lippe in der Mittellinie, der jedoch im Saume derselben narbig verheilt war. Oberhalb der Narbe aber entdeckte ich eine kleine Fistel. Eine feine Sonde lässt sich in das vordere Scheidengewölbe hinein führen, und von hier aus in die Fistel.

Aetiologisch ergiebt sich fär diesen Fall die Entstehung der Blasenfistel and Laquearfistel durch die gleiche Ursache, einen eervico-vaginalen sagittalen medianen Riss an der Vorderwand, dessen nicht von selbst verheilte Ueberreste heute sich als die beiden erwähnten Fisteln darstellen.

Diesen 19 Fällen können wir heute noch 2 neuere Beobachtungen hinzufügen:

Fall 20. Torggler beschreibt aus der seiner Zeit Sehauta'schen Klinik in Innsbruck (a. a. O. S. 198 u. 199) folgenden Fall unter Nr. 352, 1884: „M. M., 38 Jahre, hat vor zehn Wochen von selbst nach $21 / 2$ tägiger Geburtsarbeit geboren. Bald nach der Geburt vollständige Incontinenz. Sehr kurze Scheide, an vielen Stellen narbig: verengt. Im unteren Drittel der vorderen Scheidenwand grosse Fistel, halbmondförmig, $81 / 2 \mathrm{~cm}$ lang und $11 / 2 \mathrm{~cm}$ breit. Oberer Wundrand glatt, beweglich, der untere gewulstet. Harnröhre fast ganz zerstört, der etwa $22 \mathrm{~mm}$ lange Rest ist nur für die feinste Sonde durchgängig. Die vordere Muttermundslippe, theilweise zerstört, zeigt eine kleine Utero-vaginalfistel: also Blasenscheidenfistel mit Utero-vaginalfistel zugleich. Die Utero-vaginalfistel wurde nach Anfrischung durch vier Bozeman'sche Nähte geschlossen, die Blasenscheidenfistel gesondert operirt.

Nachdem bereits die Handschrift der vorliegenden Mittheilung in den Druck gegeben war, erschien ein für mich doppelt interessanter Aufsatz von Nordmann in diesem Archive (Bd. XXXIX, Hft. 1, S. 33), der einen der obigen Beobachtung von Cervico-vaginalfistel sehr ähnlichen Fall beschreibt:

Fall 21. Frau A. N. G., 32 Jahre, hat vor $4^{1 / 4}$ Jahren, $3 / 4$ Jahre nach der Hochzeit, im siebenten Monate abortirt ohne Besonderheiten im weiteren Verlaufe; vor $3 \frac{1}{2}$ Jahren wiederum siebenmonatlicher Abort, ohne jede Untersuchung der Scheide glatt verlaufen und ohne Nachkrankheiten; die Fran will jedoch gefühlt haben, als sei das Kind auf einem anderen Wege ausgetreten, als das erste Mal. Der Gatte war vier Jahre vor der Hochzeit, also vor jetzt neun Jahren, syphilitisch geworden. 
Im April 1889 wandte sich die Kranke wegen eitrigen Scheidenausflusses an Dr. Nordmann, der einen fünfzigcentimesstïckgrossen Defect in der hinteren Cervicalwand entdeckte, der äussere Muttermund bildet ein rundliches, für die Sonde durchgängiges Grübchen ohne sichtbare Narbenbildung. Der in der Mittellinie gelegene Defect der hinteren Cervicalwand, gegen die Oeffnung des Canales hin trichterförmig sich verengend, hat sammetartig weiche Ränder. Schleimhaut ringsherum normal. Der Defect in der hinteren Cervicalwand, stellt sich nach Entfernung des ihn bedeckenden schleimig-eitrigen Belages dar als eine hochrothe, leicht blutende Geschwürstläche, die sich allseitig nahezu $1 \mathrm{~cm}$ weit über den hinteren äusseren Theil des Gebärmutterhalses aus. dehnt, weiter als man dem Gefühle nach zuerst vermuthet hätte." Am übrigen Scheidentheile, sowie in den Anhängen der Gebärmutter, in der Scheide keinerlei Besonderheiten. Nordmann schwankte in der ätiologischen Deutung dieser Cervixoperation zwischen Carcinom und Syphilis. Prof. Fehling trug ringsherum die Geschwürsränder $a b$, frischte also die Fistel an und vernähte sie mit sechs Nähten in Sim s'scher Seitenlage. Das Mikroskop konnte weder Carcinom, noch Syphilis nachweisen, wohl aber ,papilläre $W$ ucherungen, die auf einen im Ausheilen begriffenen Process hinwiesen". Die Wunde heilte nicht, also zweite Anfrischung, diesmal Silberdraht. Abermals unvollkommene Heilung, es bleibt jedoch nur eine erbsengrosse granulirende Stelle noch in der Narbe, Sonde geht durch den Muttermund leicht in die Gebärmutterhöhle, ohne sichtbar zu werden in dem nachgebliebenen Fistelchen. Geheilt entlassen.

Ein Jahr später sah Nordmann die Kranke; es ist ein etwa $4 \mathrm{~mm}$ breiter, kurzer, mit Epithel überkleideter Fistelgang nachgeblieben, Ausfluss gering. Frau gesund.

Bildung eines Geschwüres an der hinteren Fläche der Vaginalportion bezw. Cervix, das sich sowohl flächenhaft als in die Tiefe ausbreitet, und eine Cervico-vaginalfistel folgt bei einer 32 jährigen Frau mit Vitium cordis, die, an einen syphilitischen Mann verheirathet, zwei Mal siebenmonatlich abortirt hat bei sehr engem Muttermunde: „Perforationsgeschwür der hinteren Wand des Scheidentheiles in dem Cervicalcanale."

Nordmann schliesst ätiologisch Syphilis, Carcinom (Tuberculose?), Abscess per exclusionem aus und denkt infolge der nach zwei Frühgeburten doch noch so auffallend engen Beschaffenheit des äusseren Muttermundes an eine Geburtsläsion, und zwar entweder durch Drucknekrose sub partu mit ihren Folgen, durch quere Cervixruptur bei engem Muttermunde oder durch eine Vereinigung beider Momente, am wahrscheinlichsten erscheint Nordmann eine Querruptur der Cervix als Ursache. Die Geschwürsbildung wäre dann eine secundäre, gerade so wie bei Emmet'schen Längenrissen, vielleicht Ulcus puerperale traumaticum? Die Ursache der Querruptur würde Nordmann vermuthen in der 
Unnachgiebigkeit des Scheidentheiles der damals 28jährigen Ipara, vielleicht spielte auch bei Starrheit der Cervix die Syphilis eine Rolle.

Nordmann beruft sich auf die in der Literatur erwähnten Fälle von Querriss der Cervix bei Unnachgiebigkeit, unter anderen auf den Piering'schen Fall aus Schauta's Prager Klinik, wo bei Fehlgeburt im sechsten Monate die Frucht durch einen hinteren Querriss ausgestossen wurde.

Nordmann hat in der Literatur keinen Fall von hinterer Cervico-vaginalfistel finden können. Nun, in meiner Fistelarbeit im vorigen Jahre habe ich $13 \mathrm{Fälle}$ von Cervico-vaginalfisteln erwähnt und jetzt abermals 8, zusammen 21. Bei entsprechender Nachlese dürfte die Zahl sich wohl schnell vergrössern, indem nach unserem Materiale von Fisteln etwa 3 Proc. der Blasengenitalfisteln von Cervicovaginalfisteln begleitet werden.

Wir sehen aus der obigen, vorläufig kleinen Casuistik von 21 Fällen von Laquearfisteln der Cervix einmal, dass dieselben in jedem Abschnitte der Vaginalportion, also in der Vorderlippe sowohl, als in der Hinterlippe vorkommen, in der letzteren überwiegend häufiger, dass sie nicht immer in der Mittellinie, sondern auch seitlich gelegen sein können, und dass sie ätiologisch meist aufzufassen sind als offen gebliebene Reste theilweise verheilter Cervical- bezw. cervico-vaginaler Risse, beziehungsweise cervicovagino-vesicaler Risse. Viel seltener, aber doch wahrscheinlich, ist die Entstehung durch instrumentelle Verletzung, möglich auch die Entstehung durch einfache, zu lange dauernde Abquetschung der Muttermundslippe mit nachfolgender Druckgangrän. Endlich zeigt sich, dass diese Laquearfisteln als Begleiterscheinungen anderer wichtigerer puerperalen Verletzungen der Cervix keineswegs selten sind, - die grösste Mehrzahl mag wohl unbemerkt bleiben und von selbst heilen. Endlich betreffen diese Beobachtungen, wie ja selbstverständlich, meist nur Frauen mit engem Becken, Dystokia durch räumliches Missverhältniss. Ist die Vermuthung Nordmann's begründet von der Verursachung einer Cervico-vaginalfistel durch primäre pathologische Unnachgiebigkeit der Cervix, so steht bislang seine Beobachtung einzig da.

Was den Fall 14 (12) anbetrifft, so ist ja leider seine Aetiologie nicht zweifellos festgestellt, jedenfalls scheint sie aber eine unmittelbar traumatische $\mathrm{zu}$ sein durch eine Verletzung von a ussen her, also eine andere, als bei den meisten anderen 
Fällen, wo die Bedingungen zur Verletzung in den anatomischen Verhältnissen, bezw. dem räumlichen Missverhältnisse zwischen mütterlichem Becken und Kind gegeben sind, sobald nicht rechtzeitig Kunsthülfe eintritt. Erfolgten zweite Schwängerung und der neue Abort hier durch die Cervixfistel? Ich glaube es und auch, dass der Abort eine Folge des Bestehens der grossen Cervicalfistel und des secundären Cervixkatarrhes gewesen ist.

Die Casuistik der Vesico-uterinfisteln ist somit heute auf 193 Fälle (31 Fälle aus der Warschauer gyniatrischen Universitätsklinik meines verstorbenen Vaters) angewachsen, die Zahl der Cervixlaquearfisteln (4 fremde und 17 Fälle aus meines Vaters und meinem Materiale) auf 21. Die Zahl der letzteren dürfte sich leicht verzehnfachen, sobald die Aufmerksamkeit der Fachgenossen hierauf gerichtet sein wird.

\section{Nachtrag zu dem Literaturverzeichnisse über vesicale Cervical- fisteln des Uterus.}

Mac Arthur: Ureteral fistulae etc. Philadelphia medical and surgical Reporter 1889, Bd. LXI, p. 225.

Baumm: Zur Operation der Blasen-Cervixisteln von der Blase aus. Siehe dieses Archiv, Bd. XXXIX, S. 492.

Boulton, siehe Champneys.

Champneys: A new operation for the cure of Vesico-Uterine Fistula. Obst. Society of London. The British medical Journal 1888, IL, p. 818. Siebe: Frommel's Jahresbericht für das Jahr 1888, II. Jahrgang.

Sherwood Dunn: Nouveau traitement chirurgical des maladies inflammatoires des reins et des uretères chez la femme. Paris 1889.

Engström: Fall of vesicouterovaginalfistel. Finska Läkaresăllskapets Handl. 1889, Bd. XXXI, No. 4, p. 344-348.

Heinricius: Treatment des fistules vésico-vaginales par la méthode américaine. Nouv. Archives d'Obstétrique et de Gynécologie 1889, p. 55-85. Johnson: British medical and surgical Journal 1889 , Bd. CXX, p. 309. More Madden: Traitement of vesicovaginal and vesicouterine Fistulae. Academy of Medicin in Ireland. Lancet 1887, I, p. 1133.

Mittelhäuser: Ueber Incontinentia urinae des Weibes und deren Behandlung. Inaugural-Dissertation. Jena 1889.

$\mathrm{N}$ agel: Die Wendung bei engem Becken. Dieses Archiv, Bd. XXXIV, Hft. 1, S. 31. Fall 57 (Maassen).

Novaro, siehe Vincenzo.

Stumpf, siehe Frommel's Jahresbericht 1887, 1888, 1889.

Morel Terry: Parto distócio: fistuia vesiconterina; curácion par la posición y el cateterismo repetido. Gac. med. de Granada 1887, p. 545.

Tillaux: De l'occlusion du vagine dans le cas de fistule vésico-vaginale. Gazette de Gynécologie 1887, p. 196.

Vincenzo: Fistula atero-cervico-vaginalis. Syringokleisis immediata. Sanatio completa. Riforma medica 1889, p. 204.

Winckel: Ueber Vesico-uterinfisteln. Sitzungsberichte der Gesellschaft für Geburtshülfe und Gynäkologie in München 1889, Hft. 2, S. 57.

Wölfler: Zur operativen Behandlung der Urinfisteln (Blasen-, Scheiden-, Gebärmutterfisteln). Bericht über die Verhandlungen des XVI. Congresses der deutschen Gesellschaft für Chirurgie im Centralblatt für Chirurgie, S. 95. 